\title{
SULLA STRUTTURA DELLA CROSTA TERRESTRE NEL MEDITERRANEO CENTRO-OCCIDENTALE E NELL'ADRIATICO
}

\author{
Paolo Emilio Valle
}

Premessa. - In una nota precedente $\left(^{1}\right)$ è stato segnalato che la velocità di gruppo media delle onde superficiali $M$ che si propagano attraverso il Meriterraneo centro-occidentale è, a parità di periodo, sensibilmente più elevata della velocità di gruppo media delle analoghe onde che si propagano attraverso il continente europeo.

Per interpretare questo fatto è stato ritenuto sufficientemente approssimato il modello di un mezzo ad una semplice stratificazione. Sotto questa ipotesi ed assumendo la velocità media delle onde traversali nel primo strato pari a $3,3 \mathrm{~km} \mathrm{sec}-1$, la curva di dispersione delle onde superficiali $M$ calcolata da Jeffreys ( ${ }^{2}$ ), ha condotto ad attribuire allo spessore medio dello strato superiore della crosta terrestre o "strato del granito ", un valore di 14-15 km nel Mediterraneo centro-occidentale ed un valore che si aggira intorno ai $25 \mathrm{~km}$, in corrispondenza del continente europeo.

I dati sperimentali da cui derivano questi risultati sono però alquanto grossolani e troppo scarsi, e quindi è stata fatta la riserva di condurre ulteriori ricerche.

In questa nota viene riportato uno studio sulla propagazione delle onde' superficiali, originate dal terremoto Jonico del 22 aprile 1948, lungo tragitti mediterranei e continentali. Viene tentata inoltre una interpretazione dei risultati, la quale è in buon accordo con lo studio preliminare a cui si è accennato.

La misura della velocitì di gruppo delle onde superficiali. - La misura della velocità di gruppo delle onde superficiali, relativa ad un certo periodo, viene eseguita dividendo la distanza epicentrale per un tempo $t$. Recentemente l'Autore ha sviluppato una teoria, che consente di dedurre rigorosamente il tempo $t$ dalla conoscenza dell'andamento della registrazione, della perturbazione iniziale e delle costanti strumentali.

Non è qui il caso di riportare la teoria, la quale peraltro è stata 


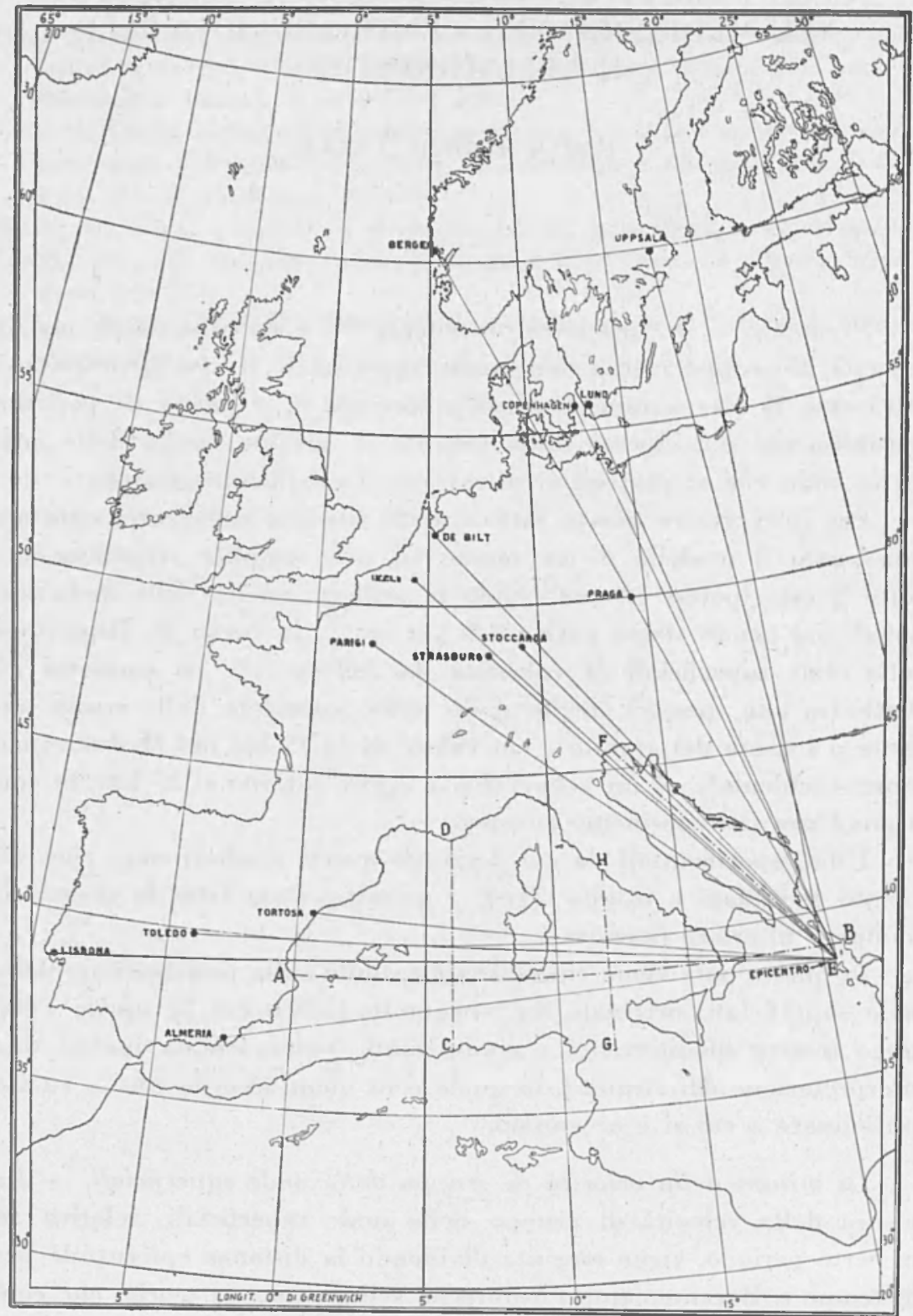

Fig. 1 
già esposta abbastanza dettagliatamente $\left({ }^{3}\right)$, basterà ricordare che se si approssima il sismogramma con sinusoidi, si ottengono notevoli semplificazioni. E facile vedere in questa approssimazione che, se il gruppo delle onde superficiali non è eccessivamente disperso e ci si limita alla misura della velocità di gruppo relativa al periodo della sinusoide per il quale il prodotto del periodo stesso per l'ampiezza è sensibilmente più grande di tutti gli analoghi prodotti che si possono eseguire nel gruppo, il tempo $t$ si ottiene, con buona approssimazione, sottraendo il tempo origine al tempo di arrivo del centro della sinusoide. Se poi il gruppo si può approssimare con più sinusoidi dello stesso periodo e ampiezza, il tempo $t$ è dato dal tempo di arrivo del centro del gruppo diminuito del tempo origine.

\section{ALMERIA}

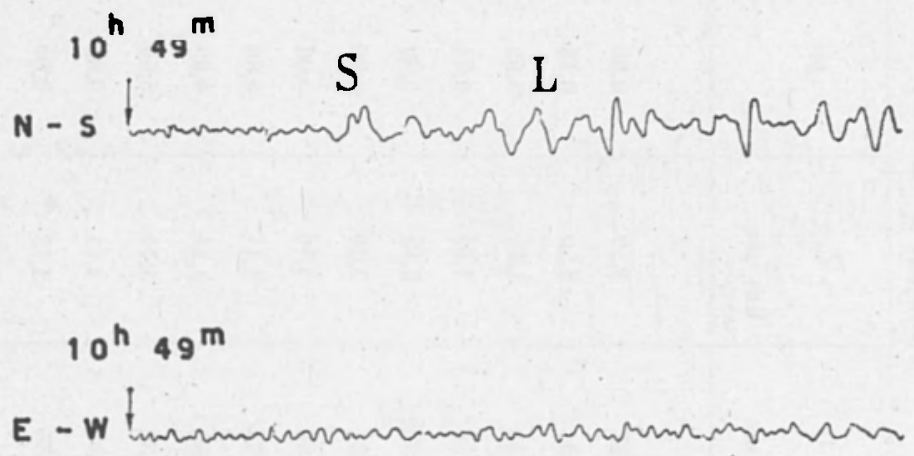

Fig. 2

Ciò vale soltanto se si trascura l'azione dello strumento e se la durata della perturbazione iniziale è trascurabile rispetto alla durata del passaggio dell'onda per il punto in cui viene effettuata la registrazione; in caso contrario sarà necessario apportare le dovute correzioni. In genere però tali correzioni sono trascurabili.

Velocità di gruppo media delle onde L nel Mediterraneo centrooccidentale, nell'Europa e nel Mar Adriatico, in corrispondenza del periodo $\mathrm{T}=20^{\mathrm{s}}$. - Lo studio della propagazione delle onde superficiali del terremoto Jonico del 22 aprile 1948, è stato limitato alle onde trasversali tangenziali $L$, le quali, data la loro natura, richiedono, per essere individuate, buone registrazioni delle sole componenti orizzontali dello spostamento.

Nella quasi totalità dei sismogrammi usati, queste onde si sono 


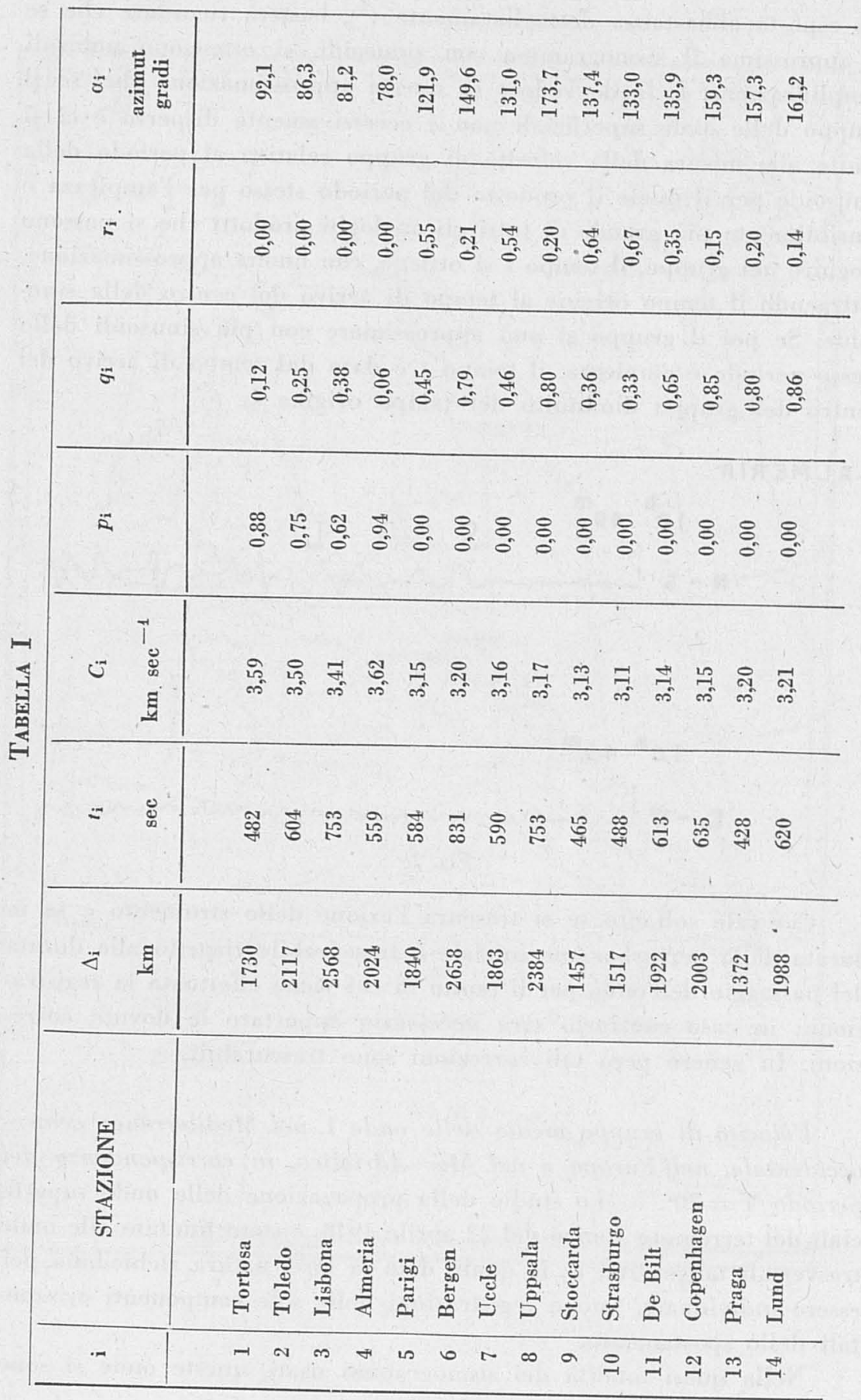


presentate notevolmente sviluppate e con le caratteristiche richieste per la misura sufficientemente corretta e spedita del tempo $t$, in corrispondenza del periodo $T=20^{\mathrm{s}}$.

Le figg. 2 e 3 mostrano due esempi di registrazioni in osservatori con azimut, rispetto all'epicentro, prossimo a $\pi / 2$. Dato che le costanti strumentali delle componenti orizzontali sono quasi uguali, la polarizzazione è evidente.

In una precedente nota $\left({ }^{4}\right)$ è stato accennato che il centro di

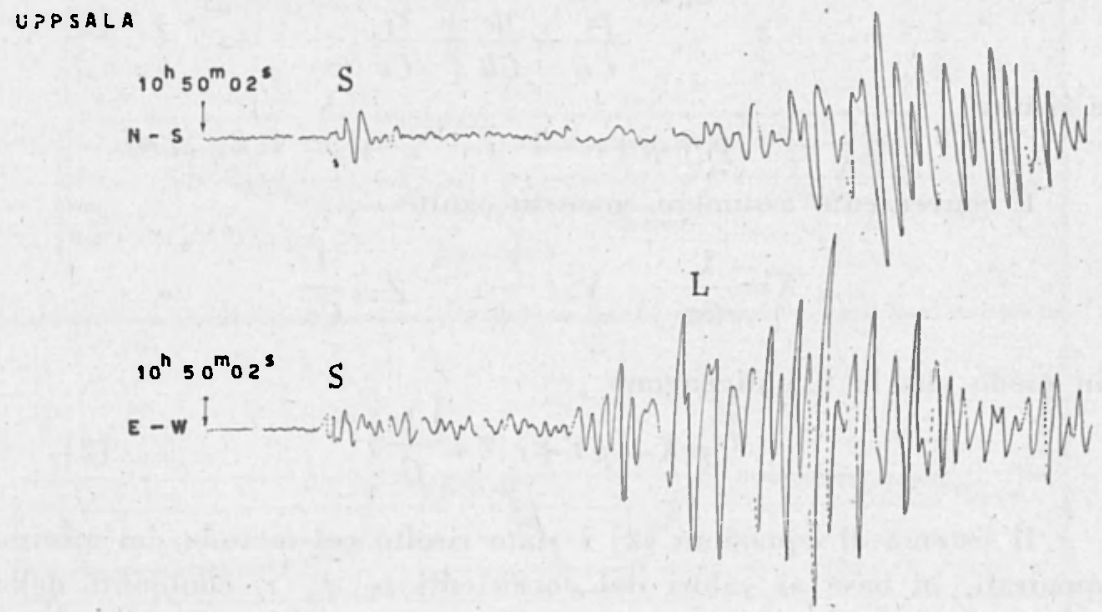

Fig. 3

perturbazione ̀̀ poco profondo e sono state calcolate le coordinate epicentrali e il tempo origine, pervenendo al seguente risultato:

$$
\begin{aligned}
& \lambda_{0}=20^{\circ}, 48+0^{\circ}, 04 \\
& \varphi_{0}=38^{\circ}, 49 \div 0^{\circ}, 04 \quad \text { (geografica) } \\
& H_{0}=10^{\mathrm{h}} 42^{\mathrm{m}} 40^{\mathrm{s}}, 8+0,4 \quad \text { (T.M.G.) }
\end{aligned}
$$

In base a questi elementi e ai tempi $t_{\mathrm{i}}$ misurati nei sismogrammi, sono state dedotte le velocità di gruppo medie $C_{\mathrm{i}}$ in 14 stazioni. I valori ottenuti sono riportati nella tabella $I$, mentre la fig. 1 mostra la distribuzione geografica delle stazioni e le geodetiche che le collegano all'epicentro.

Per calcolare da questi primi dati la velocità media di gruppo nel Mediterraneo centro-occidentale e nell'Europa, sono state valutate le percentuali dei tragitti nelle due zone, tenendo conto che parte dei tragitti interessano il Mare Adriatico e, in misura però molto minore, il Mare del Nord. 
Pertanto sono state considerate tre zone: a) Mediterraneo centrooccidentale; b) Europa; c) Mare Adriatico (e Mare del Nord).

La parte del tragitto relativo alla stazione di Parigi, che interessa l'Italia centro-meridionale ̀̀ stata assegnata alla zona $c$.

Se si indicano per ogni stazione con $p_{k} q_{k}$, ed $r_{\mathrm{i}}$ le percentuali dei tragitti rispettivamente nelle zone $a, b, c$, con $C a, C b, C c$, le relative velocità medie di gruppo e con $n$ il numero delle stazioni, si ha

e risulta

$$
C_{\mathrm{i}}=\frac{1}{\frac{\mu_{\mathrm{i}}}{r_{a}}+\frac{q_{\mathrm{i}}}{C b}+\frac{r_{\mathrm{i}}}{C c}}
$$

$$
p_{\mathrm{i}}+q_{\mathrm{i}}+r_{\mathrm{i}}=1 \quad(i=1,2, \ldots, n)
$$

E conveniente assumere come incognite

$$
X=\frac{1}{C a} \quad, \quad Y=\frac{1}{c b}, \quad Z=\frac{1}{C c}
$$

in modo che le [1] divengono

$$
p_{\mathrm{i}} X+q_{\mathrm{i}} Y+r_{\mathrm{i}} Z=\frac{1}{C_{\mathrm{i}}}
$$

Il sistema di equazioni [2] è stato risolto col metodo dei minimi quadrati, in hase ai valori dei coefficienti $p_{i} q_{i}, r_{i}$ contenuti nella tabella I.

I valori approssimati più probabili delle incognite, con $\mathrm{i}$ rispettivi errori medi, sono $i$ seguenti

$$
\begin{aligned}
& X=0,2756+0,0015 \\
& Y=0,3125+0,0015 \\
& Z=0,3232+0,0024
\end{aligned}
$$

Pertanto le velocità medie di gruppo delle onde $L$, relative al periodo $T=20^{\text {s }}$, valgono:

Mediterraneo centro-occidentale $\quad C a=3,63+0,02 \mathrm{~km} \mathrm{sec}^{-1}$

Europa $\quad C b=3,20 \pm 0,01_{\overline{5}} \mathrm{~km} \mathrm{sec}^{-1}$

Mare Adriatico (e Mare del Nord) $C c=3,09+0,02 \mathrm{~km} \mathrm{sec}^{-1}$

L'effetto della rifrazione sui valori delle grandezze $C_{i}, p_{1}, q_{i}$ ed $r_{i}$ si può ritenere trascurabile.

Infatti per i tragitti attraverso l'Adriatico, il continente e il Mare del Nord, l'indice di rifrazione è molto prossimo all'unità, come può verificarsi con $i$ dati del prossimo paragrafo, mentre per i tra- 
gritti attraverso il Mediterraneo e il continente, sebbene l'indice di rifrazione sia meno prossimo all'unità, gli angoli d'incidenza non sono troppo grandi e la percentuale del tragitto marino prevale in media largamente sulla percentuale del tragitto continentale.

Tentativo di prospezione profonda. - I valori della velocità di

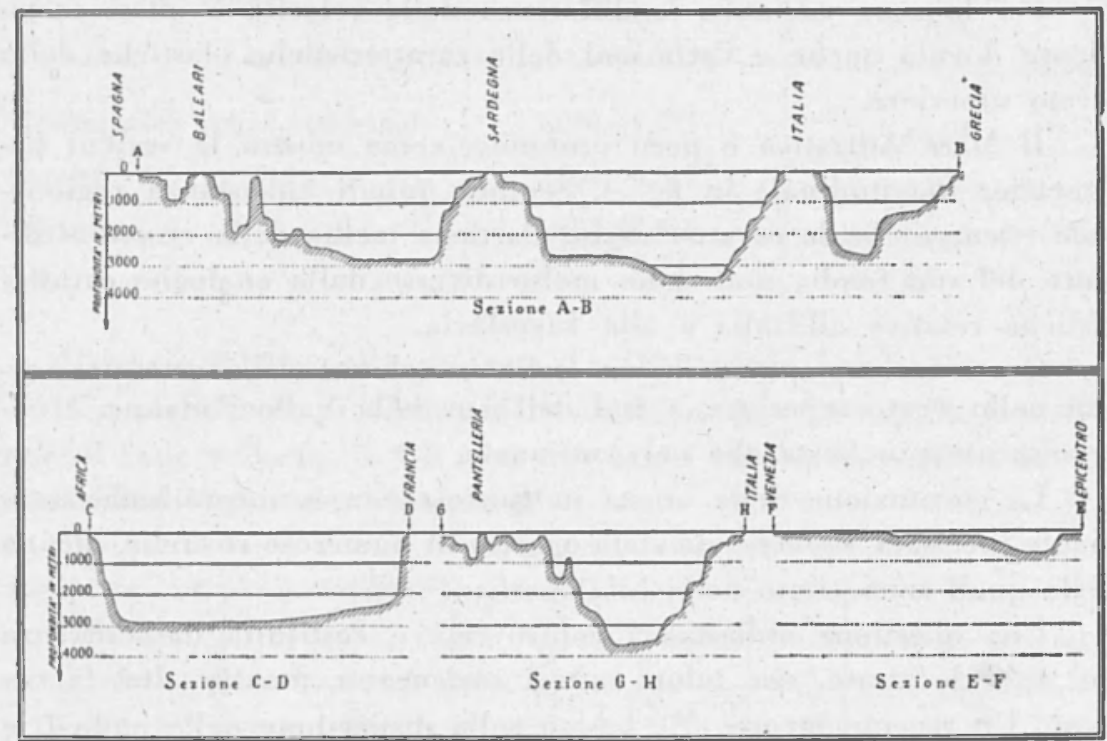

Fig. 4

gruppo delle onde $L$ nelle tre zone, mostrano che la relativa costituzione della crosta terrestre è abbastanza differente.

Vi è una certa ambiguità nell'interpretazione di questo fatto, anche perché non sono note le velocità delle onde trasversali spaziali $S g$ e $S^{*}$ nelle due zone marine.

$\mathrm{Si}$ può supporre che gli strati superiori abbiano spessori diversi nelle tre zone, oppure che abbiano insieme spessori e caratteristiche elastiche diverse.

Per quanto riguarda il Mediterraneo centro-occidentale, ̀̀ opportuno rilevare che le aliquote mediterranee dei tragitti sono state valutate in superficie, mentre, come mostrano le sezioni batimetriche approssimate in fig. 4 , le zolle continentali si estendono sensibilmente anche al disotto della superficie del mare. Sembra perciò plausibile 
attribuire l'aumento medio della velocità delle onde $L$ ad un assottigliamento medio del primo strato.

Più dettagliate informazioni si sarebbero potute avere misurando la velocità di gruppo in funzione della profondità media dei vari tratti dei tragitti delle onde superficiali nel mare, ma una misura del genere avrebbe richiesto un elevato numero di registrazioni relative alla penisola Iberica.

Per la zona adriatica la differenza della velocità è quasi certamente dovuta anche a variazioni delle caratteristiche elastiche dello strato superiore.

Il Mare Adriatico è poco profondo, come mostra la sezioni batimetrica longitudinale in fig. 4. Sembra quindi abbastanza ragionevole ritenere che le caratteristiche elastiche medie della crosta al disotto del suo fondo, non siano molto diverse dalle analoghe caratteristiche relative all'Italia e alla Jugoslavia.

Ora è ben noto $\left(^{5-7}\right)$ che in Italia la velocità delle onde trasversali nello strato superiore, a Sud dell'asse della Valle Padanna, è notevolmente più bassa che nel continente.

La costituzione della crosta in Europa non è ancora sufficientemente precisata, sebbene sia stata oggetto di numerose ricerche, alcune delle quali sono citate nella bibliografia $\left({ }^{8-13}\right)$.

Una questione abbastanza controversa è costituita dall'esistenza del secondo strato, che taluni autori sostengono, mentre altri la negano. Un recente lavoro $\left({ }^{13}\right)$, basato sulla dispersione delle onde $L$ e sui dati dedotti dai terremoti profondi, porta alla conclusione che nell'Eurasia lo spessore del primo strato è circa $33+3 \mathrm{~km}$, mentre lo spessore del secondo strato è pressoché nullo.

Tenendo conto delle considerazioni che sono state esposte e nell'ipotesi che l'azione del secondo strato sulla propagazione delle onde $\boldsymbol{L}$ sia trascurabile, si può valutare lo spessore del primo strato nelle tre zone. $\dot{E}$ chiaro che il risultato della valutazione avrà soltanto un carattere indicativo.

Per il continente ed il Mediterraneo conviene assumere come velocità delle onde $S g$ il valore medio nel primo strato, trovato per l'Europa centrale $\left({ }^{11}\right)$, ossia $3,33 \mathrm{~km} \mathrm{sec}{ }^{-1}$, il cui errore medio è molto

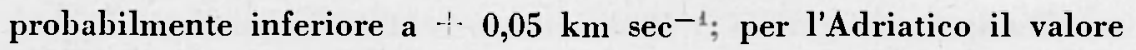
$3,01+0,02 \mathrm{~km} \mathrm{sec}^{-1}$, che costituisce il risultato della più recente misura nell'Italia centrale $\left(^{14}\right)$.

Mediante la curva di dispersione, calcolata con l'ipotesi che il rapporto tra le densità dello strato superiore e del mezzo sottostante 
sia $4 / 5$ e il rapporto delle rigidità pari a $9 / 20\left({ }^{15-16}\right)$, si ottengono per lo spessore $D$ dello strato superiore nelle tre zone $i$ valori contenuti nella tabella II.

\section{Tabella II}

\begin{tabular}{|c|c|c|}
\hline Zone & $\frac{C}{V S g}$ & $\begin{array}{c}\mathrm{D} \\
\mathbf{K} \mathbf{m}\end{array}$ \\
\hline Mediterraneo centro occidentale & $1,090+0,017$ & $15 \div 1$ \\
\hline Europa . . . . . . . . . & $0,961+0,015$ & $(30)$ \\
\hline Adriatico . . . . . . . & $1,027+0,010$ & $17+1$ \\
\hline
\end{tabular}

Il valore relativo al continente è molto incerto perché il rapporto $\mathrm{Cb} / \mathrm{VSg}$ conduce in prossimità del minimo della curva di dispersione, come si vede nella fig. 5 . Gli altri due valori sono invece affetti da un errore medio piuttosto piccolo.

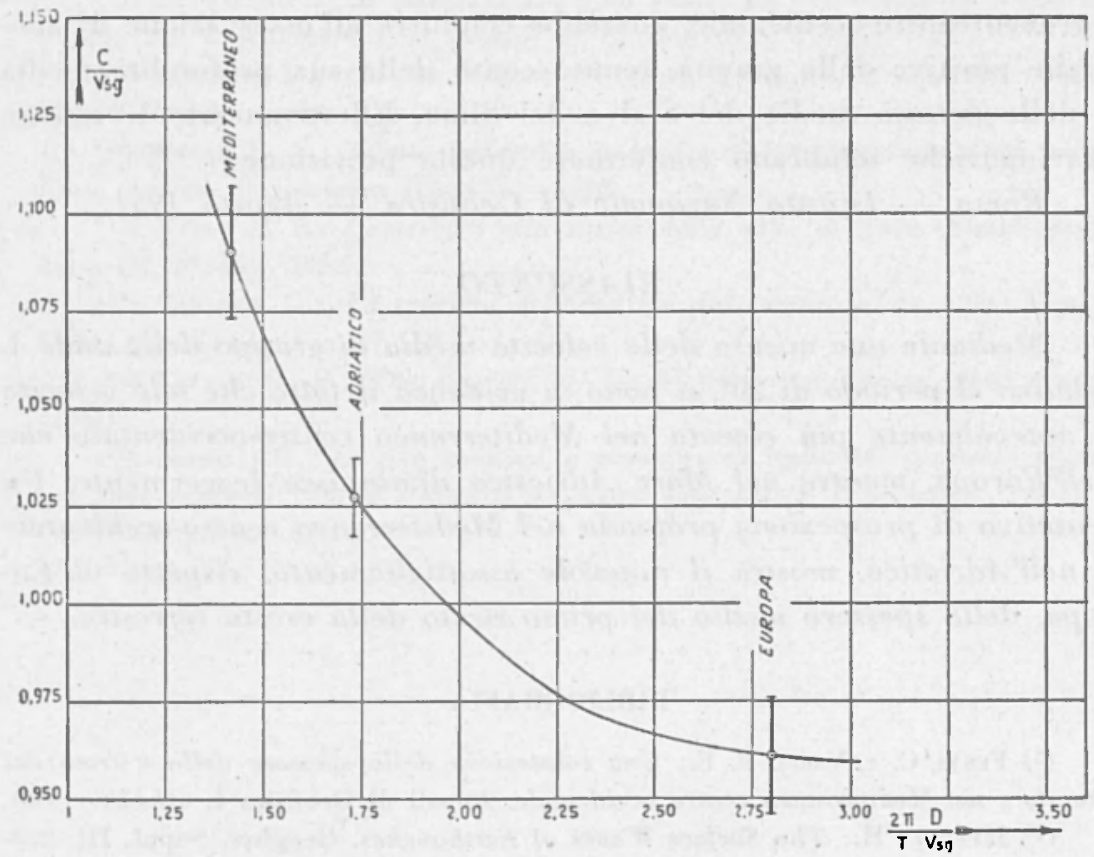

Fig. 5 
Nel quadro del modello assunto e dei valori delle grandezze fisiche che lo definiscono, è possibile avere un'idea dell'effetto dello strato sedimentario. Tale effetto si dovrà ritenere tanto più grande, quanto maggiore è la percentuale della densità dell'energia associata all'onda, in prossimità del periodo $T=20^{\mathrm{s}}$ e per gli spessori calcolati, che si propaga nello strato superiore. Sostituendo i valori dei rapporti fra le velocità di gruppo e le velocità delle onde trasversali della tabella II nelle relazioni contenute in una teoria precedentemente sviluppata dall'Autore ( ${ }^{17}$ ), risulta che la percentuale suddetta è circa il $40 \%$ per il Mediterraneo, l' $86 \%$ per il continente e il $56 \%$ per l'Adriatico.

Pertanto è da ritenere che per la zona adriatica e mediterranea l'effetto dello strato sedimentario non sia stato sensibile. Una misura della velocità di gruppo eseguita su onde di periodo maggiore, sarebbe stata certo più conveniente, ma le dimensioni delle tre zone sono troppo limitate perché la dispersione e l'assorbimento possano met. tere in evidenza onde di periodo molto lungo.

E opportuno rilevare infine, senza peraltro entrare in una dettagliata discussione, la quale potrà eventualmente essere fatta in altra sede, che l'assottigliamento medio dello strato mono denso nel Medi. terraneo centro-occidentale, dovrebbe condurre all'osservazione di anomalie positive della gravità, tenuto conto della sua profondità media e delle densità medie del Sial e del Sima. Effettivamente le misure gravimetriche sembrano confermare questa previsione $\left.{ }^{(18-20}\right)$.

Roma - Istituto Nazionale di Geofisica - Agosto 1951.

\section{RIASSUNTO}

Mediante una misura della velocità media di gruppo delle onde $\mathrm{L}$ relativa al periodo di $20^{\mathrm{s}}$, si pone in evidenza il fatto che tale velocità è notevolmente più elevata nel Mediterraneo centro-occidentale che nell'Europa, mentre nel Mare Adriatico diminuisce leggermente. Un tentativo di prospezione profonda nel Mediterraneo centro-occidentale e nell'Adriatico, mostra il notevole assottigliamento, rispetto all'Europa, dello spessore medio del primo strato della crosta terrestre.

\section{BIBLIOGRAFIA}

(1) Festa C. e Vatre P. E.: Una valutazione dello spessore dello «strato del granito 》 nel Mediterraneo centro occidentale. Annali di Geofisica I, $616-620$ (1948).

(2) JEFFreys H.: The Surface Waves of Earthquakes. Geophys. Suppl. IlI, 253 261 (1935). 
(3) Valle P. E.: Sulla misura della velocità di gruppo delle onde sismiche superficiali. Annali di Geofisica II, 370-376 (1949).

(4) Valle P. E.: Il terremoto Jonico del 22 aprile 1948. Annali di Geofisica IV, 241.246 (1951).

(5) Calor P.: Caratteristiche sismiche dell Appennino tosco-romagnolo. Ric. Scient. I, 218-234 (1940).

(6) Rosinı E.: Il terremoto della Garfagnana del 15 ottobre 1939. Ric. Scient. 7-8, 496.517 (1940).

(7) Giongi M. e Valle P. E.: Contributo allo studio delle onde ( $M$ ». Annali di Geofisica I, 87-102 (1948).

(8) Gutenberc B. and Richter C. F.: Structures of the Crust. Continents and Oceans. Physics of the Earth. VII (1939).

(9) Gutenberg B.: Seismological Evidence for roots of Mountains. Bull. Geol. Soc. Am. 54, 473-498 (1943).

(10) Jefrreys H.: A further Study of Near Earthquake. Geophys. Suppl. IV, 196-225 (1937).

(11) Calor P.: Caratteristiche sismologiche fondamentali dellEuropa Centrale quali risultano dallo studio di 17 terremoti centro.europei. Boll. Soc. Sism. Ital. XI, 41.72 (1942).

(12) Calor P.: Sullo spessore dello strato delle onde Pg nell'Europa Centrale. Ric. Scient. I, 334-338 (1938).

(13) Stoneley R.: The Continental Layers of Europe. Bull. Seismol. Soc. Am. 38, 263-274 (1948).

(14) Di Filippo D. e Marcelli L.: Uno studio sul terremoto del Gran Sasso d'ltalia del 5 settembre 1950. Annali di Geofisica IV, 213-240 (1951).

(15) Jefrreys H.: On the Surface Waves of Earthquakes. Geophys. Suppl. I, 282-292 (1925).

(16) VAlLE P. E.: Effetti dell'attrito interno sulla propagazione delle onde di Love. Annali di Geofisica III, 47-62 (1950).

(17) VAl.Le P. E.: Contributo allo studio delle onde di Love. Annali di Geofisica III, 231-250 (1950).

(18) Cassinis G.: La crociera gravimetrica del sommergibile "Des Geneys». Pubbl. Ist. Geodesia n. 47 (1935).

(19) Coster H. P.: The Gravity of the Western and Central Mediterranean. (1945).

(20) Morell C.: La rete geofisica e geodetica in Italia nel suo stato attuale e nei suoi rapporti con la struttura geologica superficiale e profonda. Pubbl. Ist. Naz. Geof. n. 121 (1946). 